\title{
Ovotesticular Disorder of Sexual Development and Non-Palpable Testis
}

\author{
Radek Štichhauer ${ }^{1, *}$, Antonín Šafus ${ }^{1}$, David Neumann², Ivo Novák ${ }^{3}$, Vladana Skutilová ${ }^{4}$, Jan Laco ${ }^{5}$
}

\begin{abstract}
Disorders of sexual development (DSD) refers to a group of diseases that links the mismatch between an individual's genetic and gonadal development and its phenotype. Ovotesticular DSD (true hermaphroditism) is one such disease, in which both male and female gonads are present.

A 15-year-old boy with a history of surgery for non-palpable testis was examined due to bilateral gynecomastia and known gonosomal mosaic of Klinefelter syndrome. The external genital was matured as male and, in the left half of the scrotum, there was a testicle of normal size. Despite uncertain resistance on the right side, however, the right testis was not palpable. Revision of the right groin revealed a surprising finding in the form of an ovary with a dilated fallopian tube, both of which were completely removed. Surgical revision of the left testis with biopsy was performed. The surgery was completed with a bilateral mastectomy. The postoperative course was uncomplicated, and the boy is content and fully integrated into his peer group. True hermaphroditism is a rare type of DSD. In the case described, DSD was not exhibited until puberty, after an examination for gynecomastia. The case also confirms the necessity of clarification and long-term follow-up of patients with unclear findings during surgery for non-palpable testis. Diagnostic laparoscopy is clearly indicated in these situations.
\end{abstract}

\section{KEYWORDS}

true hermaphroditism; ovotesticular DSD; gynecomastia; non-palpable testis; laparoscopy

AUTHOR AFFILIATIONS

${ }^{1}$ Department of Pediatric Surgery, University Hospital Hradec Králové, Faculty of Medicine Hradec Králové, Charles University, Czech Republic

${ }^{2}$ Department of Pediatrics, University Hospital Hradec Králové, Faculty of Medicine Hradec Králové, Charles University, Czech Republic

${ }^{3}$ Department of Urology, University Hospital Hradec Králové, Faculty of Medicine Hradec Králové, Charles University, Czech Republic

${ }^{4}$ Department of Medical Genetics, University Hospital Hradec Králové, Faculty of Medicine Hradec Králové, Charles University, Czech Republic

${ }^{5}$ Fingerland Department of Pathology, University Hospital Hradec Králové, Faculty of Medicine Hradec Králové, Charles University, Czech Republic

* Corresponding author: Department of Pediatric Surgery, University Hospital Hradec Králové, Sokolská 581, 50005 Hradec Králové, Czech Republic; e-mail: radek.stichhauer@fnhk.cz

Received: 1 June 2020

Accepted: 24 October 2020

Published online: 14 April 2021

Acta Medica (Hradec Králové) 2021; 64(1): 42-45

https://doi.org/10.14712/18059694.2021.7

(c) 2021 The Authors. This is an open-access article distributed under the terms of the Creative Commons Attribution License (http://creativecommons.org/licenses/by/4.0), which permits unrestricted use, distribution, and reproduction in any medium, provided the original author and source are credited. 


\section{INTRODUCTION}

Disorders of sexual development (DSD), previously termed "intersex", refers to a heterogeneous group of diseases that links the mismatch between an individual's genetic and gonadal development and their phenotype. A basic genetic status of "XX" primarily determines female genital organs and phenotype. A system of genes, namely, TDF (testis determining factor) and SRY (sex-determining region on Y-chromosome) located on the short arm of the Y chromosome, is required for male development. The presence of these genes leads to differentiation of originally undifferentiated gonads. The hormones produced by the fetal testicle then modify phenotypic sex $(1,2)$. However, there are numerous deviations and pathologies that can completely disrupt this process. Ovotesticular DSD (previously termed "true hermaphroditism") is such an example of a DSD in which both male and female gonads are present in an individual. Gonadal or phenotypic sexual disorder may occur in an individual immediately after birth; however, there are also variants that manifest later in adolescence, as in the case described in the present article, or during adulthood. Treatment of DSD involves an interdisciplinary approach, including pediatric, endocrine, surgical, urological and, finally, psychosocial care. The timing of surgery depends on the type of disorder, age at diagnosis, and psychosocial considerations (3). Given the hormonal production and the increased risk for malignant germ cell tumors in abnormally localized gonads, surgical treatment should not be postponed. At this point, DSD treatment is intertwined with treatment of non-palpable or pathologically undescended testes in otherwise healthy boys.

\section{CASE REPORT}

A 15-year-old boy was referred to the pediatric surgeon for bilateral gynecomastia and confirmed rare gonosomal mosaic of Klinefelter syndrome 46XX[84]/47XXY[16], previously examined by an endocrinologist. The patient was otherwise healthy, with normal height and body proportions. He had an unremarkable medical history, except for surgery for the presumed undescended right testis at 15 months of age. Symmetrical bilateral gynecomastia grade II was present and pubic hair within the context of pubertal development was evident (Fig. 1). The external genitalia were fully matured in the male line, Tanner G5, P4, A3. The left half of the scrotum contained a normal size testicle. The right side, however, exhibited elastic resistance in the groin extending into the scrotum, measuring approximately $2 \times 2 \mathrm{~cm}$ in area; nevertheless, the right testis was not palpable. Laboratory investigations revealed a hypergonadotropic status testosterone level, although other biochemical examinations were within reference limits. Human chorionic gonadotropin and alpha-fetoprotein levels were unremarkable. With these results, the boy was referred to an outpatient department of Pediatric Surgery. According to the currently defunct operative protocol used to revise the right groin at the age of 15 months, it was found that tissue that was supposed to be in the right testicle rudiment was removed. Diagnostic laparoscopy was not performed at that time. However, microscopic examination of the removed tissue did not confirm the presence of testicular tissue. Subsequently, the boy was lost to surveillance and follow-up. At 15 years of age, the boy underwent ultrasonography after physical examination. Normal homogeneous testis on the left and a structure mimicking a small testicle on the right, with possible incipient hydrocele, were evident. After multidisciplinary board consultation, surgery was scheduled. The actual surgery was made two months after the first examination. The resistance in the right groin was already twice as large and visible.

First, diagnostic laparoscopy was performed with the patient under general anesthesia to assess the type of internal genital organs in the abdominal and pelvic cavities. Entrances to both inguinal canals were closed. The vas deferens and spermatic vessels entered the left inguinal canal, whereas vas deferens was absent on the right side,

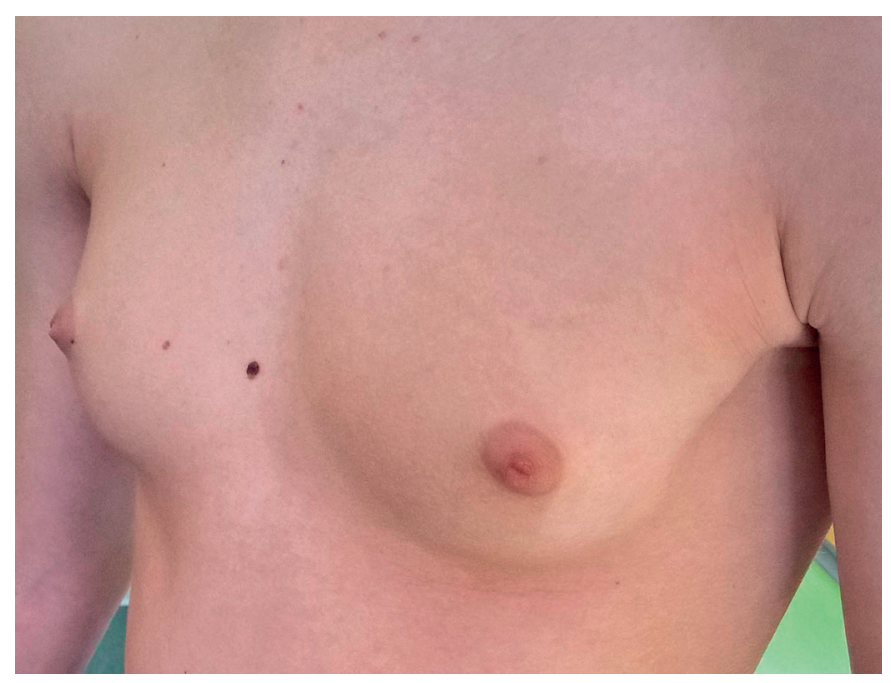

Fig. 1 Gynecomastia grade II on both sides.

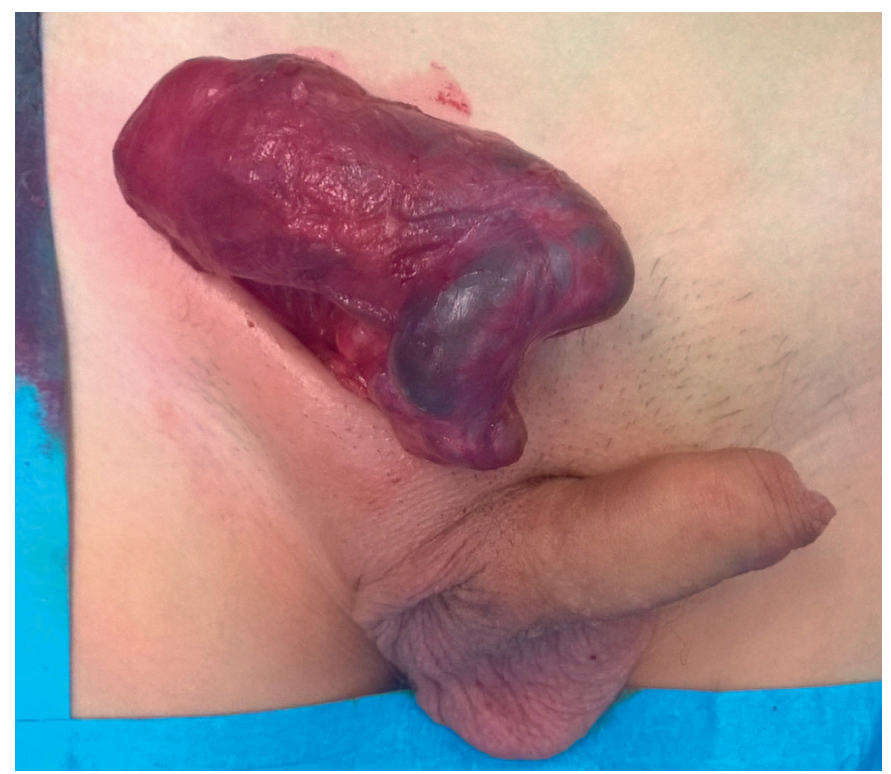

Fig. 2 Peroperative finding in the inguinal region - ovary and fallopian tube on the right. 

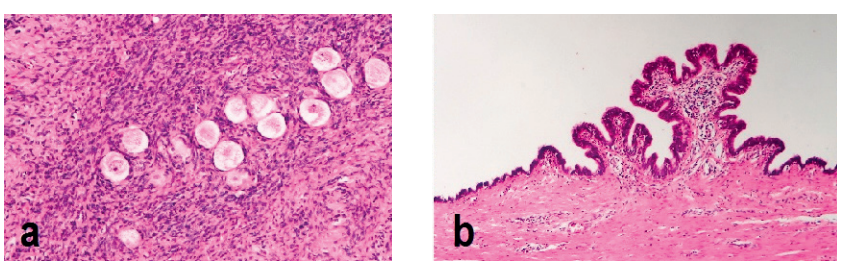

where only spermatic vessels entered the inguinal canal. Revision of the right inguinal region revealed a surprising finding of an ovary with a dilated Fallopian tube filled with serous fluid (Fig. 2); both of these organs were completely removed. Subsequently, surgical revision of the left, macroscopically normal, testis with a punch biopsy was performed. The testis was then fixed into the scrotum and has been left in situ. The surgery was completed with bilateral mastectomy, with a subareolar incision at the end of the procedure.

Microscopic examination of the tissue from the right groin revealed a fully developed but dilated fallopian tube and ovarian tissue with primordial follicles. Testicular tissue was absent. Examination of the left-side testis revealed complete sclerosis in approximately one-half of the tubules and absence of spermatogenesis in the remaining tubules. This finding was consistent with the diagnosis of germ cell aplasia. Within the interstitium, there were a few scattered cells with eosinophilic cytoplasm, most likely representing immature Leydig cells (Fig. 3).

The postoperative course was uncomplicated, and all surgical wounds healed per primam. The cosmetic outcomes in all regions were favorable. The boy is under continual care of a pediatric endocrinologist and a pediatric surgeon. His psychological well-being is favorable, and he does not need the specialized care of a mental health professional. He was fully satisfied with the postoperative outcomes and has fully integrated into his peer group.

\section{DISCUSSION}

DSD is a group of diseases manifesting as various abnormalities of genetic, gonadal, and phenotypic sex. The most common example of DSD is congenital adrenal hyperplasia, accounting for approximately $70-80 \%$ of cases. As surgical treatment, feminizing genitoplasty is usually performed. Ovotesticular disorders account for approximately $3-10 \%$ of DSDs, the most common of which include karyotype $46 \mathrm{XX}(53 \%)$, and chromosomal mosaicisms $(40 \%)$ and $46 \mathrm{XY}(7 \%)$. According to Matsui (4), the most common gonadal combination is ovotestis with ovary $(33.9 \%)$, followed by ovary and testis $(24.2 \%)$, bilateral ovotestis (20.6\%), and ovotestis and testis (16.4\%). The true hermaphrodite, an individual with a simultaneously developed ovary and testicle and karyotype $46 \mathrm{XX}, 47 \mathrm{XXY}$, as in our case, is a very rare finding. Comprehensive studies are rare, and very few have described a large number of patients. Thus, extensive experience with treatment and long-term follow-up is lacking (3). There is a $2.6-4.6 \%$ increased risk for malignant transformation of ovotesticular gonads in boys with karyotype $46 \mathrm{XY}$ (5). In girls with ovotesticular disorders, the presence of testis leads to

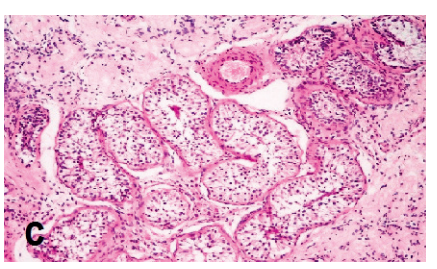

Fig. 3 Histological findings: (a)

ovary, (b) Fallopian tube, (c) testis.

early virilization; therefore, early surgical and endocrinological treatments are recommended (2). Other factors, such as age at diagnosis and psychosocial aspects, also play an important role. The majority of DSD defects are detected early after birth or in the first years of a child's life. Experience with the boy in our case and some other publications demonstrate that ovotesticular disorder may only become evident also later, in older children or even in adults. In a previous study, Caputo reported a case involving a 53-year-old man with hypergonadotropic hypogonadism that was revealed incidentally when the patient was treated for urolithiasis. Clinically, the man had micropenis, undifferentiated external organs, and normally formed secondary sexual characteristics. After genetic and magnetic resonance examination, he underwent laparoscopy with removal of a uterus with adnexa, ovary, and testis from the abdominal cavity $(6,7)$. The timing of surgery also involves psychosocial considerations. Gonadal organs are diverse in newborns or young children with ovotesticular disorders, and assessment of further development can be difficult. Previous publications have recommended postponement of definitive surgery until the pre-pubertal or adolescent period (1). In girls with karyotype $46 \mathrm{XX}$, however, there is agreement regarding the elimination of male or atypical gonads (testis, ovotestis) at an early age $(8,9)$.

The boy described in the present case, points out one more aspect, which is the suitability of diagnostic laparoscopy in the treatment of undescended and non-palpable testes. Cryptorchidism (i.e., impaired testicular descent and position in boys), is one of the most common congenital malformations in general. It affects approximately $1-4.6 \%$ of newborns but up to $30-45 \%$ of preterm newborns. At 1 year of age, the percentage of persistent cryptorchidism varies from $0.8-1.8 \%(1,2,10)$. Neonatal germ cells are converted in spermatogonia between months 3 and 9 , and there is clear evidence of a decrease in germ cells after this period and fibrous rebuilding in pre-school age. The incidence of malignant germ cell tumors in undescended testes has been reported to be approximately $1.7 \%$ (10). For these reasons, cryptorchidism should be diagnosed before 6 months of age and surgery should be performed within the first year of life. Non-palpable testes (i.e., testes that are not palpable in the scrotum or inguinal region), have been described in $10-15 \%$ of cryptorchidism cases $(10,11)$. Clinical examination is usually followed by ultrasonography (USG). While the specificity of USG examination is $91 \%$ for testes located in the inguinal canal, the sensitivity is only $78 \%$, and the yield of USG examination decreases significantly to approximately $30-40 \%$ in cases involving intra-abdominal retention (non-palpable testes) (10). In Europe, USG is used to diagnose non-palpable testes in approximately 
$49 \%$ of cases; in the United States, however, this figure is only $12 \%$ (12). Independently, USG examination is recommended as the next step in examining the patient under general anesthesia at the beginning of the operation. Currently, diagnostic laparoscopy is considered to be the gold standard modality in the treatment of non-palpable testis (12). Both, monocentric and multicentric studies describe ovotesticular disorders as an indication for gonadal exploratory diagnostic laparoscopy in cases of non-palpable testis $(11,13)$. In our case, diagnostic laparoscopy for non-palpable testis was not performed at 15 months of age, and the result of histological examination of the removed tissue from the right groin, where the testis was absent, was not correctly interpreted by the clinicians. At present, diagnostic laparoscopy is currently performed at our department in every patient with non-palpable testis or non-specific resistance in the scrotum or groin. This structure could be mistaken for atrophic testicular rudiment and the testicle itself could be located intra-abdominally. The preoperative ultrasound examination is not considered decisive. The final diagnosis is always ensured by histology. In case of unclear peroperative findings, histological examination can be performed peroperatively. Patients after these operations are observed annually until puberty.

\section{CONCLUSION}

True hermaphroditism is a rare type of ovotesticular DSD. In our case, it was not evident until puberty, following an examination for gynecomastia. After multidisciplinary examinations, surgery was performed and the ovary and fallopian tube were removed, with a histologically confirmed second testis fixed and mastectomy performed. The postoperative course was uncomplicated, and the boy is healthy, content, and fully integrated into his peer group. This case confirms the necessity of clarification and longterm follow-up of patients with unclear findings during surgery for non-palpable testis. Diagnostic laparoscopy is clearly indicated in all of these situations.

\section{ACKNOWLEDGEMENTS}

The authors are supported by the program PROGRES Q40/11 and by the project BBMRI-CZ LM2018125.

The authors thank the patient and his parents for granting permission to publish data and photographs.

\section{ABBREVIATIONS}

DSD disorders of sexual development

USG ultrasonography

\section{AUTHOR CONTRIBUTIONS}

SR designed the study and drafted the manuscript, and SR, SA, and NI performed the surgical procedures. ND performed pediatric and endocrinological examination of the patient and leads his follow-up; SV performed the biogenetics examination, and LJ performed histological examination. All authors revised and edited the draft, and are in agreement with the content of the manuscript submitted for publication.

\section{AVAILABILITY OF DATA AND MATERIALS}

All data are available upon reasonable request from the Department of Pediatric Surgery, University Hospital Hradec Králové and Charles University, Faculty of Medicine Hradec Králové, Czech Republic, Sokolska 581, 50005 Hradec Králové, Czech Republic.

\section{CONFLICT OF INTEREST STATEMENT}

The authors have no conflicts of interest regarding publication of this article.

\section{REFERENCES}

1. Skaba R. Intersex. In: Snajdauf J, Skaba R, et al. Dětská chirurgie. Praha: Galen, 2005: 373-4.

2. Gatti JM. Disorders of sexual differentiation. In: Ashcraft's Pediatric Surgery sixth edition. Toronto: Elsevier Saunders 2014: 826-37.

3. Scarpa M-G, Lesma A, Di-Grazzia M, Rigamonti W. Ovotesticular differences of sex development: male or female? Case series. Ital J Pediatr 2019; 46: 66-7.

4. Matsui F, Simada K, Matsumoto F et al. Long-term outcome of ovotesticular disorder of sex development: a single centre experience. Int $J$ Urol 2011; 18: 231-6.

5. Pleskacova J, Hersmus R, Oosterhuis JW, et al. Tumor risk in disorders of sex development. Sex Dev 2010; 4(4-5): 259-69.

6. Caputo M, Mele C, Zavattaro M, Sama MT, et al. Ovotesticular disorder of sex development: a rare case of lateral subtype $45 \mathrm{X} / 46 \mathrm{XY}$ karyotype diagnosed in adulthood. Urology 2019; 129: 68-70.

7. Berhan Y, Lemma BE, Ergete W, Gemechu T. True hermaphrodite: very unusual type. Ethiop Med J 2004; 42(3): 221-8.

8. Kilberk MJ, McLoughlin M, Pyle LC, Vogiatzi MG. Endocrine management of ovotesticular DSD, an index case and rewiew of literature. Pediatr Endocrinol Rev 2019; 17(2): 110-6.

9. Sircili MH, Denes FT, Costa EM, et al. Long-term follow up of a large cohort of patients with ovotesticular disorder of sex development. J Urol 2014; 191(Suppl 5): 1532-6.

10. Sepulveda X, Egana PJL. Current management of non-palpable testes: a literature review and clinical results. Transl Pediatr 2016; 5(4): 233-9

11. Topuzlu Tekant G, Emir H, Eroglu E, Akman M, Buyukunal C, et al. Experience with laparoscopy in nonpalpable testis. EurJ Pediatr Surg 2001; 11(3): 177-81.

12. Mah LW, Durbin-Johnson B, Kurzock EA. Non-palpable testis: in management consistent and objective? J Pediatr Urol 2020; 16(1): 62-68.

13. Abolyosr A. Laparoscopic versus open orchidopexy in the management of abdominal testis: a descriptive study. Int J Urol 2006; 13(11): 1421-4. 